\title{
Linear conformable differential system and its controllability
}

\author{
Awais Younas ${ }^{1}$, Thabet Abdeljawad ${ }^{2,3,4^{*}} \mathbb{E}$, Rida Batool ${ }^{1}$, Anum Zehra ${ }^{5}$ and Manar A. Alqudah ${ }^{6}$
}

\section{"Correspondence:}

tabdeljawad@psu.edu.sa

${ }^{2}$ Department of Mathematics and

General Sciences, Prince Sultan

University, Riyadh, Saudi Arabia

${ }^{3}$ Department of Medical Research,

China Medical University, Taichung 40402, Taiwan

Full list of author information is available at the end of the article

\begin{abstract}
This article deals with the sequential conformable linear equations. We have focused on the solution techniques of these equations and particularly on the controllability conditions of the time-invariant system. For the controllability conditions and results, we have defined the conformable controllability Gramian matrix, the conformable fundamental matrix, and the conformable controllability matrix.
\end{abstract}

Keywords: Conformable differential systems; Controllability; Time-invariant; Gramian matrix

\section{Introduction}

Fractional calculus has gained importance due to the adequate analysis approach in real world problems. It is generally used in medical sciences [27], material sciences, fluid mechanics, edge detection, and electromagnetics [12, 16, 25, 26]. Numerous physical phenomena having memory and hereditary characteristics can be efficiently interpreted through the fractional calculus approach [21]. There are various publications which are based upon the study of fractional differential equations and have also highlighted their respective applications, see [4-6, 19, 23, 24].

There are various real world problems which are expressed as both linear and nonlinear system of fractional order differential equations. The solution technique, along with the applications of such systems, can be seen in $[12,14,20]$.

Several definitions of fractional order derivative are introduced by famous researchers like Euler, Fourier, Abel, Sonin, Letnikov, Laurent, Nekrasov, and Nishimoto. Also, the most popular definitions for it are Riemann-Liouville, Caputo, and Grünwald-Letnikov definitions. Some other definitions for fractional derivatives and fractional integrals are also provided in $[16,19]$ by Kilbas and Miller.

Here are some of the well-known definitions on which we have focused throughout in our article:

- Riemann-Liouville derivative:

$$
D_{x}^{\alpha} f(x)=\frac{1}{\Gamma(n-\alpha)} \frac{d^{n}}{d x^{n}} \int_{0}^{x}(x-t)^{n-\alpha-1} f(t) d t, \quad n-1<\alpha \leq n
$$

(c) The Author(s) 2020. This article is licensed under a Creative Commons Attribution 4.0 International License, which permits use, sharing, adaptation, distribution and reproduction in any medium or format, as long as you give appropriate credit to the original author(s) and the source, provide a link to the Creative Commons licence, and indicate if changes were made. The images or other third party material in this article are included in the article's Creative Commons licence, unless indicated otherwise in a credit line to the material. If material is not included in the article's Creative Commons licence and your intended use is not permitted by statutory regulation or exceeds the permitted use, you will need to obtain permission directly from the copyright holder. To view a copy of this licence, visit http://creativecommons.org/licenses/by/4.0/. 
- Caputo derivative:

$$
D_{x}^{\alpha} f(x)=\frac{1}{\Gamma(n-\alpha)} \int_{0}^{x}(x-t)^{n-\alpha-1} f(t)^{(n)} d t, \quad n-1<\alpha \leq n
$$

- Grünwald-Letnikov derivative:

$$
{ }_{a} D_{x}^{\alpha} f(x)=\lim _{h \rightarrow 0} h^{-\alpha} \sum_{j=0}^{\left[\frac{x-a}{h}\right]}(-1)^{j}\left(\begin{array}{c}
\alpha \\
j
\end{array}\right) f(x-j h) .
$$

A few properties of these fractional order derivatives are similar to those of the classical order derivative. However, there are few drawbacks, e.g., $D_{a}^{\alpha}(1)=0$ does not fulfill in Riemann-Liouville derivative. And for Caputo derivative, we have to assume that $f$ is differentiable, otherwise we cannot apply this definition. It is also important to note that Liouville's theorem in fractional case does not hold as well. In short, all fractional derivatives are deficient in some mathematical properties, like the product, chain, and quotient rules. For a review about various properties of these fractional order derivatives, one can see [26].

Due to these issues, a new definition of fractional order derivative is required, which can fulfill more mathematical conditions as compared to the previous ones. Recently, Khalil and Horani have introduced a new well-defined and simple definition of fractional order derivative known as conformable fractional derivative [7, 15, 34]. This definition depends on the basic limit which is defined for classical order derivative. They have also established various properties like the product, quotient, and chain rules, and additionally mean value theorem of conformable fractional derivative. Some other new ideas on conformable derivative can be seen in $[1,2,9,11,28,30,31,33]$. However, the conformable fractional derivative is not considered to be the same as a fractional order derivative, it is a first-order derivative multiplied by an additional simple factor. Hence, this new definition appears to be a natural extension of the conventional order derivative to arbitrary order without memory affect. A recent new approach in defining fractional operators with nonsingular Mittag-Leffler kernel with memory affect can be found in [8].

With the popularity of fractional calculus approach in mathematical modeling, control theory has also utilized it in the controllability analysis. For a review on fractional controllability, one can see $[10,18,22,32]$. In these articles the controllability criterion and conditions with different fractional order derivatives are discussed.

With this motivation, in this article, we have used the definitions of conformable fractional derivative, conformable fractional integrals, and some fundamental results of conformable fractional calculus. By using the definition of conformable fractional derivative, we will demonstrate the Liouville's theorem, the controllability conditions for a timeinvariant system.

The methodology of our work in this manuscript has two strategies: the first is a direct technique and the second uses a transformation into state equations. We will also define a conformable fractional transition matrix and the fundamental matrix.

For obtaining the controllability conditions, we will study sequential linear conformable fractional differential equations of order $n \alpha$. We will revise some results about the existence and uniqueness for order $n \alpha$ sequential linear conformable fractional homogeneous 
and nonhomogeneous fractional differential equations defined in the following form:

$$
T^{n \alpha} y+a_{n-1} T^{(n-1) \alpha} y+a_{n-2} T^{(n-2) \alpha} y+\cdots+a_{0} y=0
$$

and

$$
T_{\alpha}^{n} y+p_{n-1}(t) T_{\alpha}^{n-1} y+\cdots+p_{1}(t) T_{\alpha} y+p_{0}(t) y=f(t)
$$

with

$$
y\left(t_{0}\right)=y_{0}, \quad T_{\alpha} y\left(t_{0}\right)=y_{1}, \quad \ldots, \quad T_{\alpha}^{n-1} y\left(t_{0}\right)=y_{n-1}, \quad a<t_{0}<b,
$$

respectively $[3,13]$. There are several ways to solve the conformable fractional differential equations, some of which are the same as those involving the classical order conformable derivative.

\section{Basic concepts}

Here, basic results and a few definitions on conformable fractional calculus will be revised.

Definition 1 ([1]) Let $a \in \mathbb{R}$. For a function $f:[a, \infty) \rightarrow \mathbb{R}$, the conformable fractional derivative $T_{\alpha}^{a}$ of $f$ of order $\alpha \in(0,1]$ is given by

$$
T_{\alpha}^{a} f(t)=\lim _{\varepsilon \rightarrow 0} \frac{f\left(t+\varepsilon(t-a)^{1-\alpha}\right)-f(t)}{\varepsilon} \text { for all } t>a .
$$

In particular, if $a=0$ then (4) becomes

$$
T_{\alpha} f(t)=\lim _{\varepsilon \rightarrow 0} \frac{f\left(t+\varepsilon t^{1-\alpha}\right)-f(t)}{\varepsilon} .
$$

If a given function $f$ satisfies Definition 1 for all $t>a$, then $f$ is called an $\alpha$-differentiable function.

Lemma 1 ([15]) Let $f, g:[a, \infty) \rightarrow \mathbb{R}$ be $\alpha$-differentiable functions at a point $t>a$, then

1. $T_{\alpha}^{a}(f g)=f T_{\alpha}^{a}(g)+g T_{\alpha}^{a}(f)$;

2. $T_{\alpha}^{a}\left(\frac{f}{g}\right)=\frac{g T_{\alpha}^{a}(f)-f T_{\alpha}^{a}(g)}{g^{2}}$, where $g \neq 0$ for all $t>a$;

3. Iff is differentiable, then

$$
T_{\alpha}^{a}(f)(t)=(t-a)^{1-\alpha} \frac{d f(t)}{d t}
$$

Remark 1 The $\alpha$-derivative is a linear operator, that is,

$$
T_{\alpha}^{a}(\lambda f+\beta g)=\lambda T_{\alpha}^{a}(f)+\beta T_{\alpha}^{a}(g) .
$$

Definition 2 ([15]) The $\alpha$-fractional integral for a function $f$, is defined as

$$
I_{\alpha}^{a}(f)(t)=\int_{a}^{t} \frac{f(x)}{x^{1-\alpha}} d x
$$

where the integral is the usual improper Riemann integral and $\alpha \in(0,1)$. 
Remark 2 The $\alpha$-fractional integral is a linear operator if $t>a$ :

$$
I_{\alpha}^{a}(\lambda f+\beta g)=\lambda I_{\alpha}^{a}(f)+\beta I_{\alpha}^{a}(g) .
$$

Lemma 2 ([15]) For any continuous function $f$ in the domain of $I_{\alpha}$ we have

$$
T_{\alpha}^{a} I_{\alpha}^{a}(f)(t)=f(t)
$$

Lemma $3([1]) I_{\alpha}^{a} T_{\alpha}^{a}(f)(t)=f(t)-f(a)$, where $f:(a, b) \rightarrow \mathbb{R}$ is an $\alpha$-differentiable function and $0<\alpha \leq 1$.

Let us consider the following conformable fractional linear homogeneous differential equation:

$$
T_{\alpha} y+p(t) y=0,
$$

and conformable fractional nonhomogeneous differential equation

$$
T_{\alpha} y+p(t) y=q(t)
$$

where $y \in C^{1}, p(t)$ and $q(t)$ are real-valued continuous functions.

If we have a conformable fractional initial value problem

$$
T_{\alpha} y+p(t) y=q(t), \quad y\left(t_{0}\right)=y_{0},
$$

where $p(t)$ and $q(t)$ are assumed to be continuous and defined for all $t \in(a, b)$, then there exists a unique solution of the initial value problem (8).

Lemma 4 ([13]) Let $t^{\alpha-1} p(t), t^{\alpha-1} q(t) \in C(a, b)$, and y be a continuously differentiable function. Then, the initial value problem

$$
T_{\alpha} y+p(t) y=q(t), \quad y\left(t_{0}\right)=y_{0},
$$

has a unique solution on the interval $(a, b)$ where $t_{0} \in(a, b)$.

Let us consider a second-order conformable fractional equation of the following form:

$$
T_{\alpha} T_{\alpha} y+a_{1} T_{\alpha} y+a_{0} y=r(t)
$$

where $a_{0}, a_{1}$ are real constants and $r(t)$ is a nonzero continuous function, and a homogeneous fractional differential equation of the form

$$
T_{\alpha} T_{\alpha} y+a_{1} T_{\alpha} y+a_{0} y=0 .
$$

Definition 3 The conformable fractional Wronskian $W_{\alpha}\left[y_{1}, y_{2}\right]$ for two independent functions $y_{1}$ and $y_{2}$ is defined as

$$
W_{\alpha}\left[y_{1}, y_{2}\right]=\left|\begin{array}{cc}
y_{1} & y_{2} \\
y_{1}^{(\alpha)} & y_{2}^{(\alpha)}
\end{array}\right| .
$$


Lemma 5 If $y_{1}$ and $y_{2}$ are two linearly independent solutions of conformable fractional homogeneous equation (11), then the particular solution $y_{p}$ of corresponding the conformable fractional nonhomogeneous equation (10) is

$$
y_{p}(x)=I_{\alpha}^{a}(K(x, t) f(t))
$$

where

$$
K(x, t)=\frac{\left|\begin{array}{ll}
y_{1}(t) & y_{2}(t) \\
y_{1}(x) & y_{2}(x)
\end{array}\right|}{W_{\alpha}\left[y_{1}, y_{2}\right](t)} .
$$

Let us consider the linear sequential conformable homogeneous fractional differential equation of order $n \alpha$ given by

$$
T_{\alpha}^{(n)} y+a_{n-1} T_{\alpha}^{(n-1)} y+a_{n-2} T_{\alpha}^{(n-2)} y+\cdots+a_{0} y=0,
$$

where the coefficients $a_{0}, a_{1}, \ldots, a_{n}$ are real constants, $\alpha \in(0,1)$ and $T_{\alpha}^{(n)}=T_{\alpha} T_{\alpha} \cdots T_{\alpha}$. If $y$ is $n$ times differentiable, then there exist $n$ independent solutions $y_{1}, y_{2}, \ldots, y_{n}$ for the homogeneous equation (13). For $\alpha=1$, equation (13) becomes an $n$th order linear homogeneous differential equation. Let us discuss the nonhomogeneous case

$$
\left\{\begin{array}{l}
T_{\alpha}^{(n)} y+a_{n-1} T_{\alpha}^{(n-1)} y+\cdots+a_{1}(t) T_{\alpha} y+a_{0}(t) y=f(t), \\
y\left(t_{0}\right)=y_{0}, \\
T_{\alpha} y\left(t_{0}\right)=y_{1}, \quad \ldots, \quad T_{\alpha}^{n-1} y\left(t_{0}\right)=y_{n-1}, \quad a<t_{0}<b .
\end{array}\right.
$$

Note that for $\alpha=1$, equation (14) becomes an $n$th order linear nonhomogeneous differential equation.

Now, we will define the conformable fractional Wronskian for the particular solution of (14).

Definition 4 ([3]) If equation (13) has $n$ linearly independent solutions $y_{1}, y_{2}, \ldots, y_{n}$ then the function

$$
W_{\alpha}\left[y_{1}, y_{2}, \ldots, y_{n}\right]=\left|\begin{array}{cccc}
y_{1} & y_{2} & \cdots & y_{n} \\
y_{1}^{(\alpha)} & y_{2}^{(\alpha)} & \cdots & y_{n}^{(\alpha)} \\
\vdots & \vdots & \ddots & \vdots \\
y_{1}^{((n-1) \alpha)} & y_{2}^{((n-1) \alpha)} & \cdots & y_{n}^{((n-1) \alpha)}
\end{array}\right|
$$

is called the conformable fractional Wronskian.

Theorem 1 ([3]) If $y_{1}, y_{2}, \ldots, y_{n}$ are linearly independent solutions of the n $\alpha$-order linear conformable fractional differential equation (13), then the particular solution for (14) is

$$
y_{p}(x)=\sum_{m=1}^{n} y_{m} \int_{a}^{x} \frac{f(t) W_{m}^{\alpha}(t)}{W^{\alpha} t^{1-\alpha}} d t
$$

here $W^{\alpha}(x)=W^{\alpha}\left(y_{1}, y_{2}, \ldots, y_{n}\right)(x)$ and $W_{m}^{\alpha}=\operatorname{det}\left(W^{\alpha}\right)$ after substituting $(0,0, \ldots, 1)$ into the mth column. 
It is important to mention here that there are some problems with the previous technique. For example, it is a fact that for complementary solutions of (13), we have to change this equation into a classical order differential equation, which becomes a lengthy process. Moreover, for the particular solution $y_{p}$ of (13) it is necessary that $y_{1}, y_{2}, \ldots, y_{n}$ are linearly independent. Here we make the following changes of variables for equation (14):

$$
x_{1}=y, \quad x_{2}=T_{\alpha} y, \quad x_{3}=T_{\alpha}^{2} y, \quad \ldots, \quad x_{n-1}=T_{\alpha}^{n-1} y .
$$

Therefore, we can write

$$
\begin{aligned}
& T_{\alpha} x_{1}=x_{2}, \\
& T_{\alpha} x_{2}=x_{3}, \\
& \cdots, \\
& T_{\alpha} x_{n-1}=x_{n}, \\
& T_{\alpha} x_{n}=-a_{n-1} x_{n}-\cdots-a_{1} x_{2}-p_{a 0} x_{1}+q(t),
\end{aligned}
$$

in the matrix form as:

$$
T_{\alpha}\left[\begin{array}{c}
x_{1} \\
x_{2} \\
\vdots \\
x_{n-1} \\
x_{n}
\end{array}\right]+\left[\begin{array}{cccccc}
0 & -1 & 0 & 0 & \cdots & 0 \\
0 & 0 & -1 & 0 & \cdots & 0 \\
\vdots & \vdots & \vdots & \vdots & \ddots & \vdots \\
0 & 0 & 0 & 0 & \cdots & -1 \\
a_{0} & a_{1} & a_{2} & a_{3} & \cdots & a_{n-1}
\end{array}\right]\left[\begin{array}{c}
x_{1} \\
x_{2} \\
\vdots \\
x_{n-1} \\
x_{n}
\end{array}\right]=\left[\begin{array}{c}
0 \\
0 \\
\vdots \\
0 \\
q(t)
\end{array}\right],
$$

so that

$$
T_{\alpha} x(t)+P(t) x(t)=Q(t)
$$

where

$$
\begin{aligned}
x(t) & =\left[\begin{array}{c}
x_{1} \\
x_{2} \\
\vdots \\
x_{n-1} \\
x_{n}
\end{array}\right], \\
P(t) & =\left[\begin{array}{cccccc}
0 & -1 & 0 & 0 & \cdots & 0 \\
0 & 0 & -1 & 0 & \cdots & 0 \\
\vdots & \vdots & \vdots & \vdots & \ddots & \vdots \\
0 & 0 & 0 & 0 & \cdots & -1 \\
a_{0} & a_{1} & a_{2} & a_{3} & \cdots & a_{n-1}
\end{array}\right],
\end{aligned}
$$


and

$$
Q(t)=\left[\begin{array}{c}
0 \\
0 \\
\vdots \\
0 \\
q(t)
\end{array}\right] .
$$

Equation (16) is called the state representation of equation (14). The choice of state variables is by no means unique. In fact, the choice is limitless. So, the existence of the solution and its uniqueness of are firstly addressed for this state equation representation.

To solve the nonhomogeneous conformable fractional vector differential equation

$$
T_{\alpha} x(t)=A(t) x+q(t)
$$

we first solve its corresponding homogeneous portion, that is,

$$
T_{\alpha} x(t)=A(t) x .
$$

For this, we need a linear conformable fractional differential operator.

Definition 5 Consider a set $A$ consisting of all $n$ by 1 continuously differentiable vectorvalued functions on a certain used interval $I$. Let $B$ be a set consisting of all $n$ by 1 continuous vector-valued functions on the same interval $I$ ( $A$ and $B$ are linear spaces). Let us define $L_{\alpha}: A \rightarrow B$ as

$$
L_{\alpha} \xi(t)=T_{\alpha} \xi(t)-A(t) \xi(t)
$$

for $t \in I$, then $L_{\alpha}$ is called a conformable fractional vector differential operator.

Lemma 6 The conformable fractional vector differential operator $L_{\alpha}$ is linear, that is,

$$
L_{\alpha}\left[\lambda_{1} x+\lambda_{2} y\right](t)=\lambda_{1} L_{\alpha}[x](t)+\lambda_{2} L_{\alpha}[y](t),
$$

where $x$ and $y$ both are $n$ by 1 continuously differentiable vector-valued functions and $\lambda_{1}$, $\lambda_{2}$ are arbitrary real constants.

Theorem 2 If $\left(\lambda_{0}, \xi_{0}\right)$ be an eigenpair for the matrix $A$ of order $n \times n$, then

$$
\xi(t)=e^{\lambda_{0} \frac{t^{\alpha}}{\alpha}} \xi_{0},
$$

is a solution of $T_{\alpha} \xi(t)=A(t) \xi$ on $\mathbb{R}$.

Proof Let us consider

$$
\xi(t)=e^{\lambda_{0} \frac{t^{\alpha}}{\alpha}} \xi_{0} .
$$


Applying the $\alpha$-conformable derivative on both sides, we have

$$
T_{\alpha} \xi(t)=e^{\lambda_{0} \frac{t^{\alpha}}{\alpha}} \lambda_{0} \xi_{0}
$$

As $\left(\lambda_{0}, \xi_{0}\right)$ is an eigenpair,

$$
e^{\lambda_{0} \frac{t^{\alpha}}{\alpha}} A x_{0}=A e^{\lambda_{0} \frac{t^{\alpha}}{\alpha}} \xi_{0}=A \xi(t),
$$

for $t \in \mathbb{R}$, and the proof is completed.

Example 1 We will solve the conformable fractional differential equation given as

$$
T_{\alpha} x=\left[\begin{array}{cc}
0 & 1 \\
-4 & -5
\end{array}\right] x
$$

The characteristic equation of $A$ can be written as

$$
\lambda^{2}+5 \lambda+4=(\lambda+1)(\lambda+4)=0
$$

and the eigenvalues are $\lambda_{1}=-1$ and $\lambda_{2}=-4$. The first eigenpair for $A$ corresponding to $\lambda_{1}=-1$ is

$$
\left(-1,\left[\begin{array}{c}
1 \\
-1
\end{array}\right]\right) \text {. }
$$

Similarly, the eigenpair for $\lambda_{2}=-4$ is

$$
\left(-4,\left[\begin{array}{c}
1 \\
-4
\end{array}\right]\right) \text {. }
$$

Hence from Theorem 2, the vector functions $\psi_{1}$ and $\psi_{2}$ are of the form

$$
\psi_{1}(t)=e^{\frac{-t^{\alpha}}{\alpha}}\left[\begin{array}{c}
1 \\
-1
\end{array}\right], \quad \psi_{2}(t)=e^{\frac{-4 t^{\alpha}}{\alpha}}\left[\begin{array}{c}
1 \\
-4
\end{array}\right] .
$$

Since $\psi_{1}$ and $\psi_{2}$ are linearly independent on $\mathbb{R}$, the general solution $x(t)$ is of the following form:

$$
x(t)=c_{1} e^{\frac{-t^{\alpha}}{\alpha}}\left[\begin{array}{c}
1 \\
-1
\end{array}\right]+c_{2} e^{\frac{-4 t^{\alpha}}{\alpha}}\left[\begin{array}{c}
1 \\
-4
\end{array}\right], \quad t \in \mathbb{R} .
$$

Let us define the matrix conformable fractional equation as

$$
T_{\alpha} X(t)=A(t) X,
$$

where both $X(t)$ and $A$ are $n$ by $n$ continuous matrix functions on an interval $I$. We say that a matrix function $\Psi$ is a solution of (19) on $I$ if $\Psi$ is a continuously differentiable $n$ by $n$ 
matrix function on $I$ such that (19) holds for all $t \in I$; and then $\Psi$ is called the conformable fractional fundamental matrix for conformable fractional vector differential equation (18) for $t \in I$.

The next theorem establishes the relationship between (18) and (19).

Theorem 3 Let $A$ be a continuous $n$ by $n$ matrix function, and $\Psi(t)$ be defined on an interval I as

$$
\Psi(t)=\left[\psi_{1}(t), \psi_{2}(t), \ldots, \psi_{n}(t)\right], \quad t \in I .
$$

Then $\Psi(t)$ is the solution of the conformable fractional differential matrix equation (19) on I if and only if each column $\psi_{1}(t), \psi_{2}(t), \ldots, \psi_{n}(t)$ is a solution of the conformable fractional vector differential equation (18) on I for $1 \leq i \leq n$. Moreover, if $\Psi(t)$ is the solution of the conformable fractional differential matrix equation (19) then $x(t)=\Psi(t)$ c is a solution of the conformable fractional differential vector equation (18) for any constant vector $c$ of order $n \times 1$.

Proof Assume that $\psi_{i}, i=1,2, \ldots, n$ are solutions of (18) on $I$. Then the $n$ by $n$ matrix function $\Psi$ is of the following form:

$$
\Psi(t)=\left[\psi_{1}(t), \psi_{2}(t), \ldots, \psi_{n}(t)\right], \quad t \in I .
$$

As $\Psi$ is a continuously differentiable matrix function, we can easily obtain $T_{\alpha} \Psi(t)=A \Psi(t)$ for $t \in I$. Hence it is proved that $\Psi$ is a solution of conformable matrix differential equation (19). Next, for the converse part, suppose that $\Psi$ is the solution of conformable matrix differential equation (19). We have to prove that $\psi_{i}$ s are solutions of (18).

As $\Psi$ is a solution of conformable matrix differential equation (19),

$$
T_{\alpha}\left[\psi_{1}(t), \psi_{2}(t), \ldots, \psi_{n}(t)\right]=\left[A \psi_{1}(t), A \psi_{2}(t), \ldots, A \psi_{n}(t)\right]
$$

This implies that

$$
\begin{aligned}
& T_{\alpha} \psi_{1}(t)=A \psi_{1}(t), \\
& T_{\alpha} \psi_{2}(t)=A \psi_{2}(t), \\
& \cdots, \\
& T_{\alpha} \psi_{n}(t)=A \psi_{n}(t),
\end{aligned}
$$

and this shows that $\psi_{i}$ s are solutions of (18). Next assume that $\Psi(t)$ is the solution of conformable fractional differential matrix equation (19) and let $t \in I$. Consider $x(t)=\Psi(t) c$. Then

$$
T_{\alpha} x(t)=A x(t)
$$

for $t \in I$, which proves the theorem. 
Theorem 4 (Fractional Liouville's theorem) If $\Psi(t)=\left[\psi_{1}(t), \psi_{2}(t), \ldots, \psi_{n}(t)\right]$ is the matrix function of $n$ solutions of the conformable differential vector equation (18), then for $t_{0} \in I$,

$$
\operatorname{det} \Psi(t)=e^{I_{\alpha}^{t_{0}} \operatorname{tr}[A(s)]} \operatorname{det} \Psi\left(t_{0}\right) .
$$

Proof We will prove this result for $n=2$. Suppose $\psi_{1}(t)$ and $\psi_{2}(t)$ are two solutions of (18) on $I$ and $\Psi$ is a matrix function of the form

$$
\Psi(t)=\left[\psi_{1}(t), \psi_{2}(t)\right]
$$

where

$$
\psi_{1}(t)=\left[\begin{array}{l}
\psi_{11}(t) \\
\psi_{21}(t)
\end{array}\right] \text { and } \quad \psi_{2}(t)=\left[\begin{array}{l}
\psi_{12}(t) \\
\psi_{22}(t)
\end{array}\right]
$$

Let

$$
h(t)=\operatorname{det} \Psi(t)=\left|\begin{array}{ll}
\psi_{11}(t) & \psi_{12}(t) \\
\psi_{21}(t) & \psi_{22}(t)
\end{array}\right|, \quad \text { for } t \in I .
$$

Taking the conformable fractional derivative of both sides, we get

$$
T_{\alpha}^{t_{0}} h(t)=\left|\begin{array}{cc}
T_{\alpha}^{t_{0}} \psi_{11}(t) & T_{\alpha}^{t_{0}} \psi_{12}(t) \\
\psi_{21}(t) & \psi_{22}(t)
\end{array}\right|+\left|\begin{array}{cc}
\psi_{11}(t) & \psi_{12}(t) \\
T_{\alpha}^{t_{0}} \psi_{21}(t) & T_{\alpha}^{t_{0}} \psi_{22}(t)
\end{array}\right|,
$$

or

$$
T_{\alpha}^{t_{0}} h(t)=\left[a_{11}+a_{22}\right]\left|\begin{array}{ll}
\psi_{11}(t) & \psi_{12}(t) \\
\psi_{21}(t) & \psi_{22}(t)
\end{array}\right|
$$

After simplifying, we have

$$
T_{\alpha}^{t_{0}} h(t)=\operatorname{tr}[A(t)] h(t)
$$

Solving this conformable differentiable equation results in

$$
T_{\alpha} h(t)-\operatorname{tr}[A(t)] h(t)=0 .
$$

Multiplying equation (20) with $e^{I_{\alpha}^{t_{0}}-\mathrm{tr}[A(t)] h(t)}=e^{-\int_{t_{0}}^{t} \frac{\mathrm{tr}[A(s)] h(s)}{s^{1-\alpha}} d s}$, we can write

$$
e^{-\int_{t_{0}}^{t} \frac{\operatorname{tr}[A(s)] h(s)}{s^{1-\alpha}} d s} T_{\alpha} h(t)-e^{-\int_{t_{0}}^{t} \frac{\operatorname{tr}[A(s)] h(s)}{s^{2-\alpha}} d s} \operatorname{tr}[A(t)] h(t)=0 .
$$

Thus

$$
T_{\alpha}\left[h(t) e^{-\int_{t_{0}}^{t} \frac{\operatorname{tr}[A(s)] h(s)}{s^{1-\alpha}} d s}\right]=0 .
$$


Applying $I_{\alpha}^{t_{0}}$ on both sides, we have

$$
h(t) e^{-\int_{t_{0}}^{t} \frac{\operatorname{tr}[A(s)] h(s)}{s^{1-\alpha}} d s}=c_{0},
$$

and at $t=t_{0}$ we can write

$$
h\left(t_{0}\right) e^{-\int_{t_{0}}^{t_{0} \operatorname{tr}[A(s)] h(s)}} \frac{s^{1-\alpha}}{s^{1-\alpha}}=c_{0},
$$

which implies that $h\left(t_{0}\right)=c_{0}$. Substituting the value of $c_{0}$ into equation (21), we get

$$
h(t) e^{-\int_{t_{0}}^{t} \frac{\operatorname{tr}[A(s)] h(s)}{s^{1-\alpha}} d s}=h\left(t_{0}\right),
$$

or $h(t)=e^{\int_{t_{0}}^{t} \frac{\mathrm{tr}[A(s)] h(s)}{s^{1-\alpha}} d s} h\left(t_{0}\right)$, which can be written as

$$
h(t)=e^{I_{\alpha}^{t_{0}} \operatorname{tr}[A(s)]} h\left(t_{0}\right), \quad \text { for } t \in I,
$$

or equivalently,

$$
\operatorname{det} \Psi(t)=e^{I_{\alpha}^{t_{0}} \operatorname{tr}[A(s)]} \operatorname{det} \Psi\left(t_{0}\right), \quad \text { for } t \in I,
$$

which completes the proof.

With the above theorem, we can write a corollary that helps us observe the behavior of $\operatorname{det} \Psi(t)$ for all $t \in I$.

Corollary 1 If $\Psi$ is the matrix function with columns $\psi_{1}(t), \psi_{2}(t), \ldots, \psi_{n}(t)$ of $n$ solutions of the conformable differential vector equation (18) on I then either

1. For all $t \in I$, $\operatorname{det} \Psi(t)=0$, or

2. For all $t \in I$, $\operatorname{det} \Psi(t) \neq 0$.

Proof There are two cases which we will study, namely, either $\psi_{1}(t), \psi_{2}(t), \ldots, \psi_{n}(t)$ are linearly independent or linearly dependent. The first case holds if and only if the solutions $\psi_{1}(t), \psi_{2}(t), \ldots, \psi_{n}(t)$ of conformable vector differential equation (18) are linearly dependent on $I$, while the second case holds if and only if the solutions $\psi_{1}(t), \psi_{2}(t), \ldots, \psi_{n}(t)$ of conformable differential vector equation (18) are lineally independent on $I$.

For case 1 , assume that $\psi_{1}(t), \psi_{2}(t), \ldots, \psi_{n}(t)$ are linearly dependent. As $\psi_{1}(t), \psi_{2}(t), \ldots$, $\psi_{n}(t)$ are linearly dependent for all $t \in I$, then they are linearly dependent for $t=t_{0}$. By the definition of linear dependence of vectors, we have

$$
c_{1} \psi_{1}(t)+c_{2} \psi_{2}(t)+\cdots+c_{n} \psi_{n}(t)=0,
$$

where not all $c_{i} \mathrm{~S}$ are zero. This is equivalent to

$$
\Psi\left(t_{0}\right) c=0, \quad \text { for } c \neq 0,
$$


implying that zero is an eigenvalue of $\Psi\left(t_{0}\right)$. As we can write the determinant of any matrix as the product of its eigenvalues,

$$
\operatorname{det} \Psi\left(t_{0}\right)=0
$$

and, by Theorem 4, we have

$$
\operatorname{det} \Psi(t)=e^{I_{\alpha}^{t_{0}} \operatorname{tr}[A(s)]} \operatorname{det} \Psi\left(t_{0}\right)=0, \quad \text { for } t \in I .
$$

Next, for the converse part, we assume, for all $t \in I$, thatdet $\Psi(t)=0$. By Theorem 4 ,

$$
e^{I_{\alpha}^{t_{0}} \operatorname{tr}[A(s)]} \operatorname{det} \Psi\left(t_{0}\right)=0, \quad \text { for all } t \in I,
$$

or equivalently,

$$
\operatorname{det} \Psi\left(t_{0}\right)=0
$$

By the fact which we have already used that the determinant of any matrix is actually equal to the product of its eigenvalues, we have zero as an eigenvalue of $\Psi$ and we can write

$$
\Psi\left(t_{0}\right) x=0, \quad \text { for } x \neq 0 .
$$

This implies that columns $\psi_{1}\left(t_{0}\right), \psi_{2}\left(t_{0}\right), \ldots, \psi_{n}\left(t_{0}\right)$ of $\Psi\left(t_{0}\right)$ are linearly dependent. As $t_{0} \in$ $I$ is arbitrary, so this part is true for all $t_{0} \in I$, proving that $\psi_{1}(t), \psi_{2}(t), \ldots, \psi_{n}(t)$ are linearly dependent for all $t \in I$.

Now if $\psi_{1}(t), \psi_{2}(t), \ldots, \psi_{n}(t)$ are linearly independent, by the definition of linear independence of vectors, we have

$$
c_{1} \psi_{1}(t)+c_{2} \psi_{2}(t)+\cdots+c_{n} \psi_{n}(t)=0 \quad \text { only when all } c_{i} \text { s are zero. }
$$

This implies

$$
\Psi(t) c=0, \quad \text { where } c \text { is a zero vector. }
$$

In (23), it is clear that $\Psi(t)$ is invertible, that is, $\operatorname{det} \Psi(t) \neq 0$. Now, for the converse part, if $\operatorname{det} \Psi(t) \neq 0$ then for all $t \in I, \psi_{1}(t), \psi_{2}(t), \ldots, \psi_{n}(t)$ are linearly independent.

Definition 6 A matrix function $\Psi_{n \times n}$ is said to be a conformable fractional fundamental matrix for the conformable fractional vector differential equation (18) if $\Psi$ is a solution of the conformable fractional matrix equation (19) on $I$ such that $\operatorname{det} \Psi(t) \neq 0$ on $I$.

Theorem 5 A matrix function $\Psi_{n \times n}$ is a conformable fractional fundamental matrix for the conformable fractional vector differential (18) if and only if the columns of $\Psi$ are $n$ linearly independent solutions of (18) on I. If $\Psi$ is a conformable fractional fundamental matrix for the conformable differential vector equation (18), then the general solution $x$ of conformable fractional vector differential equation (18) for $t \in I$ is given by

$$
x(t)=\Psi(t) c_{1},
$$


where $c_{1}$ is an arbitrary constant vector of order $n \times 1$. There are infinitely many conformable fundamental matrices for the conformable differential vector equation (18).

Proof Consider that the columns of $\Psi_{n \times n}$ are linearly independent solutions of (18) on $I$. Since the columns of $\Psi$ are the solutions of (18), then, by Theorem 3, $\Psi$ is a solution of (19). Since the columns of $\Psi$ are linearly independent solutions for (18), from Corollary (1) we have $\operatorname{det} \Psi(t)=0$ on $I$. Hence $\Psi$ is the conformable fundamental matrix for conformable differential vector equation (18).

Next, for the converse part, we assume that $\Psi$ is the conformable fundamental matrix for the conformable differential vector equation (18). Then by the definition of conformable fundamental matrix, $\Psi$ is the solution matrix of equation (19) on $I$ such that $\operatorname{det} \Psi(t) \neq 0$. From Theorem 3, columns of $\Psi$ are solutions of (18). Since det $\Psi(t) \neq 0$ and columns of $\Psi$ are solutions of (18), by Corollary 1, columns of $\Psi$ are linearly independent. Since for any nonsingular $n$ by $n$ matrix $X_{0}$, the solution of the IVP

$$
T_{\alpha} X(t)=A(t) X, \quad X\left(t_{0}\right)=X_{0}
$$

is a fundamental matrix for (18). Next assume that $\Psi$ is a conformable fractional fundamental matrix for (18). Then by Theorem 3, $x(t)=\Psi(t) c$ is a solution of (18). Now consider that $z$ is an arbitrary, but fixed solution of (18). Here we define

$$
c_{0}=\Psi^{-1}\left(t_{0}\right) z\left(t_{0}\right), \quad t_{0} \in I
$$

Then $z$ and $\Psi(t) c_{0}$ are solutions of (18) which have the same vector value at $t_{0}$. Hence, by the uniqueness theorem, we have $z(t)=\Psi(t) c_{0}$. Therefore, for $t \in I$,

$$
x(t)=\Psi(t) c,
$$

where $c$ is an arbitrary constant vector, defines a general solution of (18).

Example 2 For the conformable fractional vector differential equation

$$
T_{\alpha} x=\left[\begin{array}{cc}
-2 & 3 \\
2 & 3
\end{array}\right] x
$$

find the conformable fractional fundamental matrix $\Psi$ and write the general solution of this equation (24) in the term of $\Psi$.

Solution. For the matrix $A$, the characteristic equation is as follows:

$$
\lambda^{2}-\lambda-12=0,
$$

and the respective eigenvalues and eigenvectors are $\lambda_{1}=-3, \lambda_{2}=4,\left[\begin{array}{c}3 \\ -1\end{array}\right]$, and $\left[\begin{array}{l}1 \\ 2\end{array}\right]$. From Theorem 6 , the vector functions $\psi_{1}$ and $\psi_{2}$, defined as

$$
\psi_{1}(t)=e^{\frac{-3 t^{\alpha}}{\alpha}}\left[\begin{array}{c}
3 \\
-1
\end{array}\right] \quad \text { and } \quad \psi_{2}(t)=e^{\frac{4 t^{\alpha}}{\alpha}}\left[\begin{array}{l}
1 \\
2
\end{array}\right] \text {, }
$$


are solutions of (24), and the matrix function $\Psi(t)$, given by

$$
\Psi(t)=\left[\psi_{1}(t), \quad \psi_{2}(t)\right]=\left[\begin{array}{cc}
3 e^{-3 \frac{t^{\alpha}}{\alpha}} & e^{4 \frac{t^{\alpha}}{\alpha}} \\
-e^{-3 \frac{t^{\alpha}}{\alpha}} & 2 e^{4 \frac{t^{\alpha}}{\alpha}}
\end{array}\right]
$$

is a matrix solution of conformable fractional matrix equation corresponding to (19). Since $\operatorname{det} \Psi(t)=7 e^{\frac{t^{\alpha}}{\alpha}} \neq 0$, this implies that $\Psi$ is a conformable fractional fundamental matrix of (24) on $\mathbb{R}$. Also, from Theorem 5 , the general solution of (24) is given by

$$
x(t)=\Psi(t) c
$$

for $t \in \mathbb{R}$, where $c$ is any $2 \times 1$ constant vector.

Theorem 6 If $\Psi$ is a fundamental matrix for (18), then $\Phi=\Psi C$ is a general conformable fractional fundamental matrix of (18), where $C$ is a nonsingular constant matrix of order $n \times n$.

Proof Consider that $\Psi$ is a conformable fractional fundamental matrix for (18) and let $\Phi=\Psi C$, where $C$ is any $n \times n$ constant matrix. Then $\Phi$ is a continuously differential function on $I$ and

$$
\begin{aligned}
T_{\alpha} \Phi(t) & =T_{\alpha} \Psi(t) C \\
& =A(t) \Psi(t) C \\
& =A(t) \Phi(t) .
\end{aligned}
$$

Hence $\Phi=\Psi C$ is a solution of the conformable fractional matrix equation (19).

Now for the general conformable fractional fundamental matrix, assume that $C$ is a nonsingular matrix. Since

$$
\begin{aligned}
\operatorname{det} \Phi(t) & =\operatorname{det}[\Psi(t) C] \\
& =\operatorname{det}[\Psi(t)] \operatorname{det}[C] \\
& \neq 0,
\end{aligned}
$$

for $t \in I, \Phi=\Psi C$ is a conformable fractional fundamental matrix of (18). Now the only remaining thing to prove is that any conformable fractional fundamental matrix is of the correct form. suppose that $\Phi$ is an arbitrary, but fixed matrix of (18). Let $t \in I$ and $C_{0}=$ $\Psi^{-1}\left(t_{0}\right) \Phi\left(t_{0}\right)$. Then $C_{0}$ is a nonsingular constant matrix

$$
\Phi\left(t_{0}\right)=\Psi\left(t_{0}\right) C_{0}
$$

therefore, we have $\Phi(t)=\Psi(t) C$.

For the solution of conformable fractional time-invariant system, we need to define conformable fractional matrix exponential function and its properties. 
Definition 7 For the matrix $A$ of $n \times n$ order, the conformable fractional matrix exponential function defined by $e^{\frac{A t^{\alpha}}{\alpha}}$ is the solution of conformable fractional initial value problem

$$
T_{\alpha} X=A X, \quad X(0)=I_{n \times n} .
$$

In the following theorem we will give some properties of the conformable fractional matrix exponential function.

Theorem 7 Assume that $A$ and $B$ are $n \times n$ constant matrices. Then

1. $T_{\alpha}^{a} e^{A \frac{(t-a)^{\alpha}}{\alpha}}=A e^{A \frac{(t-a)^{\alpha}}{\alpha}}=e^{A \frac{(t-a)^{\alpha}}{\alpha}} A$.

2. $\operatorname{det}\left[e^{A \frac{(t-a)^{\alpha}}{\alpha}}\right] \neq 0$, for $t \in[a, b)$.

3. If $A B=B A$, then $e^{A \frac{(t-a)^{\alpha}}{\alpha}} B=B e^{A \frac{(t-a)^{\alpha}}{\alpha}}$, for $t \in[a, b)$.

4. If $A B=B A$, then $e^{A \frac{(t-a)^{\alpha}}{\alpha}} e^{B^{\frac{(t-a)^{\alpha}}{\alpha}}}=e^{(A+B) \frac{(t-a)^{\alpha}}{\alpha}}$.

5. $e^{A \frac{(t-a)^{\alpha}}{\alpha}}=\sum_{k=0}^{\infty} \frac{A^{k}(t-a)^{\alpha}}{\alpha^{k} k !}$.

6. If $A$ has $n$ distinct eigenvalues, say, $\lambda_{1}, \lambda_{2}, \ldots, \lambda_{n}$, then there exists a non-singular matrix $P$ such that

$$
e^{A \frac{(t-a)^{\alpha}}{\alpha}}=P \operatorname{diag}\left\{e^{\lambda_{1} \frac{(t-a)^{\alpha}}{\alpha}}, e^{\lambda_{2} \frac{(t-a)^{\alpha}}{\alpha}}, \ldots, e^{\lambda_{n} \frac{(t-a)^{\alpha}}{\alpha}}\right\} P^{-1}
$$

Proof We will show that all these properties hold, as $e^{A \frac{(t-a)^{\alpha}}{\alpha}}$ is differentiable. From Lemma 1 , it is obvious that property 1 holds. Since $e^{A \frac{(t-a)^{\alpha}}{\alpha}}$ is an identity matrix and at $t=a, \operatorname{det}(I)=1 \neq 0$, from [29, Corollary 2.24]. So, $\operatorname{det}\left[e^{A \frac{(t-a)^{\alpha}}{\alpha}}\right] \neq 0$ for all $t \in[a, b)$. Hence property 2 is satisfied. To establish property 3 , let us consider

$$
\Psi(t)=e^{A \frac{(t-a)^{\alpha}}{\alpha}} B-B e^{A \frac{(t-a)^{\alpha}}{\alpha}} .
$$

Now, when applying $T_{\alpha}^{a}$ to equation (25), we have

$$
\begin{aligned}
T_{\alpha}^{a} \Psi(t) & =e^{A \frac{(t-a)^{\alpha}}{\alpha}} A B-B A e^{A \frac{(t-a)^{\alpha}}{\alpha}} \\
& =e^{A \frac{(t-a)^{\alpha}}{\alpha}} A B-A B e^{A \frac{(t-a)^{\alpha}}{\alpha}} \\
& =A\left(e^{A \frac{(t-a)^{\alpha}}{\alpha}} B-B e^{A \frac{(t-a)^{\alpha}}{\alpha}}\right) \\
& =A \Psi(t) .
\end{aligned}
$$

So $\Psi$ becomes a solution of the conformable fractional matrix equation $T_{\alpha}^{a} X=A X$. Also $\Psi(a)=0$, so by [13, Theorem 4.2], $\Psi(t)=0$. Hence

$$
e^{A \frac{(t-a)^{\alpha}}{\alpha}} B=B e^{A \frac{(t-a)^{\alpha}}{\alpha}} .
$$

For property 4, we assume that $A B=B A$ and consider

$$
\Psi(t)=e^{A \frac{(t-a)^{\alpha}}{\alpha}} e^{B \frac{(t-a)^{\alpha}}{\alpha}}-e^{(A+B) \frac{(t-a)^{\alpha}}{\alpha}} .
$$


Then, by using equation (25),

$$
\begin{aligned}
T_{\alpha}^{a} \Psi(t) & =A e^{A \frac{(t-a)^{\alpha}}{\alpha}} e^{B \frac{(t-a)^{\alpha}}{\alpha}}+B e^{A \frac{(t-a)^{\alpha}}{\alpha}} e^{B \frac{(t-a)^{\alpha}}{\alpha}}-(A+B) e^{(A+B) \frac{(t-a)^{\alpha}}{\alpha}} \\
& =(A+B)\left[e^{A \frac{(t-a)^{\alpha}}{\alpha}} e^{B \frac{(t-a)^{\alpha}}{\alpha}}-e^{(A+B) \frac{(t-a)^{\alpha}}{\alpha}}\right] \\
& =(A+B) \Psi(t),
\end{aligned}
$$

for $t \in[a, b)$. Also, $\Psi(a)=0$, so by the uniqueness theorem [13], $\Psi(t)=0$. Hence

$$
e^{A \frac{(t-a)^{\alpha}}{\alpha}} e^{B \frac{(t-a)^{\alpha}}{\alpha}}=e^{(A+B) \frac{(t-a)^{\alpha}}{\alpha}} .
$$

We know that $e^{A t}=\sum_{k=0}^{\infty} \frac{A t^{k}}{k !}$. Replacing $t$ by $\frac{(t-a)^{\alpha}}{\alpha}$, we obtain

$$
e^{A \frac{(t-a)^{\alpha}}{\alpha}}=\sum_{k=0}^{\infty} \frac{A^{k}(t-a)^{\alpha}}{\alpha^{k} k !} .
$$

This implies that property 5 is satisfied. Property 6 can be simply proved as in the classical order case.

By using the above definition and properties, we prove the following theorem.

\section{Theorem 8 Consider fractional nonhomogeneous system}

$$
T_{\alpha}^{a} y(t)=A y(t)+f(t)
$$

where $0<\alpha \leq 1$, where $y$ and $f:[a, b) \rightarrow \mathbb{R}^{n}$ are vector functions and $A$ is an $n \times n$ constant matrix. The general solution of the fractional nonhomogeneous system is

$$
y(t)=e^{A \frac{(t-a)^{\alpha}}{\alpha}} C+\int_{a}^{t} e^{A \frac{(t-a)^{\alpha}}{\alpha}} e^{-A \frac{(s-a)^{\alpha}}{\alpha}} f(s)(s-a)^{\alpha-1} d s,
$$

where $C$ is a constant vector and

$$
e^{A \frac{(t-a)^{\alpha}}{\alpha}}=\sum_{k=0}^{\infty} \frac{A^{k}(t-a)^{k \alpha}}{\alpha^{k} k !} .
$$

Proof Here the fractional nonhomogeneous system $T_{\alpha}^{a} y(t)=A y(t)+f(t)$ can be written as

$$
T_{\alpha}^{a} y(t)-A y(t)=f(t) .
$$

Multiplying equation (27) with integrating factor $e^{-A \frac{(t-a)^{\alpha}}{\alpha}}$ on both sides, we can write

$$
T_{\alpha}^{a}\left(e^{-A \frac{(t-a)^{\alpha}}{\alpha}} y(t)\right)=e^{-A \frac{(t-a)^{\alpha}}{\alpha}} f(t) .
$$

Applying fractional integration on the both sides, we have

$$
I_{\alpha}^{a} T_{\alpha}^{a}\left(e^{-A \frac{(t-a)^{\alpha}}{\alpha}} y(t)\right)=I_{\alpha}^{a}\left(e^{-A \frac{(t-a)^{\alpha}}{\alpha}} f(t)\right) .
$$


Lemma 3 now yields

$$
e^{-A \frac{(t-a)^{\alpha}}{\alpha}} y(t)-y(a)=\int_{a}^{t} e^{-A \frac{(s-a)^{\alpha}}{\alpha}} f(s)(s-a)^{1-\alpha} d s,
$$

or

$$
\begin{aligned}
y(t) & =e^{A \frac{(t-a)^{\alpha}}{\alpha}} y(a)+\int_{a}^{t} e^{A \frac{(t-a)^{\alpha}}{\alpha}} e^{-A \frac{(s-a)^{\alpha}}{\alpha}} f(s)(s-a)^{\alpha-1} d s \\
& =e^{A \frac{(t-a)^{\alpha}}{\alpha}} C+\int_{a}^{t} e^{A \frac{(t-a)^{\alpha}}{\alpha}} e^{-A \frac{(s-a)^{\alpha}}{\alpha}} f(s)(s-a)^{\alpha-1} d s,
\end{aligned}
$$

where $C$ is a constant vector and $e^{A \frac{(t-a)^{\alpha}}{\alpha}}=\sum_{k=0}^{\infty} \frac{A^{k}(t-a)^{k \alpha}}{\alpha^{k} k !}$.

Now, we move towards the solution of the linear conformable differential equation

$$
T_{\alpha}^{t_{0}} x(t)=A(t) x(t)
$$

For this, we construct a Peano-Baker series. By the definition of conformable integral, the Peano-Baker series becomes

$$
\begin{aligned}
\Psi(t, \tau) & =I+\int_{\tau}^{t} \frac{A\left(\delta_{1}\right)}{\left(\delta_{1}-t_{0}\right)^{1-\alpha}} d \delta_{1} \\
& +\int_{\tau}^{t} \frac{A\left(\delta_{1}\right)}{\left(\delta_{1}-t_{0}\right)^{1-\alpha}} \int_{\tau}^{\delta_{1}} \frac{A\left(\delta_{2}\right)}{\left(\delta_{2}-t_{0}\right)^{1-\alpha}} d \delta_{2} d \delta_{1} \\
& +\cdots \\
& +\int_{\tau}^{t} \frac{A\left(\delta_{1}\right)}{\left(\delta_{1}-t_{0}\right)^{1-\alpha}} \int_{\tau}^{\delta_{1}} \frac{A\left(\delta_{2}\right)}{\left(\delta_{2}-t_{0}\right)^{1-\alpha}} \cdots \int_{\tau}^{\delta_{k-1}} \frac{A\left(\delta_{k}\right)}{\left(\delta_{k}-t_{0}\right)^{1-\alpha}} d \delta_{k} d \delta_{k-1} \cdots d \delta_{1} \cdots
\end{aligned}
$$

Theorem 9 For $t_{0} \in I$ and vector $x_{0}$, the conformable linear state equation (28) with continuous $A(t)$ has the unique and continuously differentiable solution

$$
x(t)=\Psi\left(t_{0}, t\right) x_{0} .
$$

If we have the equation

$$
T_{\alpha}^{t_{0}} x(t)=A(t) x(t)+B(t) F(t)
$$

where $A(t)$ and $B(t)$ are $n \times n$ matrices and $x(t), F(t)$ are $n \times 1$ vectors, then we can guess the solution of the form

$$
x(t)=\Psi\left(t_{0}, t\right) x_{0}+\int_{t_{0}}^{t} \Psi\left(t_{o}, t\right) \Psi\left(t_{0}, \delta\right) B(\delta) F(\delta)\left(\delta-t_{0}\right)^{\alpha-1} d \delta
$$

For time-invariant conformable fractional linear state equation, we can write the approximating sequence (31) as

$$
x_{0}(t)=x_{0},
$$




$$
\begin{aligned}
x_{1}(t) & =x_{0}+A I_{\alpha}^{t_{0}} x_{0} \\
& =x_{0}+A x_{0} \int_{t_{0}}^{t} \frac{d \delta_{1}}{\left(\delta_{1}-t_{0}\right)^{1-\alpha}} \\
& =x_{0}+A x_{0} \frac{\left(t-t_{0}\right)^{\alpha}}{1 ! \alpha}
\end{aligned}
$$

and

$$
\begin{aligned}
x_{2}(t) & =x_{0}+A I_{\alpha}^{t_{0}} x_{1}(t) \\
& =x_{0}+A \int_{t_{0}}^{t} \frac{\left[x_{0}+A x_{0} \frac{\left(\delta_{1}-t_{0}\right)^{\alpha}}{\alpha}\right]}{\left(\delta_{1}-t_{0}\right)^{1-\alpha}} d \delta_{1} \\
& =x_{0}+A \int_{t_{0}}^{t} \frac{x_{0}}{\left(\delta_{1}-t_{0}\right)^{1-\alpha}} d \delta_{1}+A^{2} x_{0} \int_{t_{0}}^{t} \frac{\left(\delta_{1}-t_{0}\right)^{2 \alpha-1}}{\alpha} d \delta_{1} \\
& =X_{0}+A X_{0} \frac{\left(t-t_{0}\right)^{\alpha}}{1 ! \alpha}+A^{2} X_{0} \frac{\left(t-t_{0}\right)^{2 \alpha}}{2 ! \alpha^{2}}
\end{aligned}
$$

and so on. The general term of this sequence is

$$
\begin{aligned}
x_{k}(t) & =x_{0}+A I_{\alpha}^{t_{0}} x_{k-1}(t) \\
& =x_{0}+A \int_{t_{0}}^{t} \frac{x_{k-1}\left(\delta_{1}\right) d \delta_{1}}{\left(\delta_{1}-t_{0}\right)^{1-\alpha}} \\
& =x_{0}+A x_{0} \frac{\left(t-t_{0}\right)^{\alpha}}{1 ! \alpha}+A^{2} x_{0} \frac{\left(t-t_{0}\right)^{2 \alpha}}{2 ! \alpha^{2}}+\cdots+A^{k} x_{0} \frac{\left(t-t_{0}\right)^{k \alpha}}{k ! \alpha^{2}} \\
& =\left[I+A \frac{\left(t-t_{0}\right)^{\alpha}}{1 ! \alpha}+A^{2} \frac{\left(t-t_{0}\right)^{2 \alpha}}{2 ! \alpha^{2}}+\cdots+A^{k} \frac{\left(t-t_{0}\right)^{k \alpha}}{k ! \alpha^{2}}\right] x_{0},
\end{aligned}
$$

the limit of the sequence is the familiar solution

$$
x(t)=e^{A \frac{\left(t-t_{0}\right)^{\alpha}}{\alpha}} x_{0} .
$$

Thus in this case the transition matrix is just a conformable fractional matrix exponential function. Thus if we have the system

$$
T_{\alpha}^{t_{0}} x(t)=A x(t)+B F(t)
$$

where $A, B \in \mathbb{R}^{n \times n}$ matrices and $x(t), F(t)$ are $n \times 1$ vectors. Then we can guess the solution of the form

$$
x(t)=e^{A \frac{\left(t-t_{0}\right)^{\alpha}}{\alpha}} x_{0}+\int_{t_{0}}^{t} e^{A \frac{\left(t-t_{0}\right)^{\alpha}}{\alpha}} e^{-A \frac{\left(\delta-t_{0}\right)^{\alpha}}{\alpha}} B F(\delta)\left(\delta-t_{0}\right)^{\alpha-1} d \delta .
$$

\section{Controllability}

In this section, we will discuss the concept of conformable fractional controllability of time-invariant system

$$
T_{\alpha}^{t_{0}} x(t)=A x(t)+B F(t), \quad t \in\left[t_{0}, t_{0}+\tau\right],
$$


where $x(t) \in \mathbb{R}^{n}, B \in \mathbb{R}^{n \times m}, A \in \mathbb{R}^{n \times n}$, and $F(t)$ is a control vector of order $m \times 1$. For given $t_{0}, x_{0}$, and $T>0$, we construct a sequence of $n \times 1$ vector functions $\left\{x_{k}(t)\right\}_{k=0}^{\infty}$ defined on $\left[t_{0}, t_{0}+T\right]$ which can be interpreted as a sequence of approximate solutions of the equation with $x_{0}(t)=x_{0}$,

$$
\begin{aligned}
x_{1}(t)= & x_{0}+I_{\alpha}^{t_{0}}\left(A(t) x_{0}(t)\right) \\
= & x_{0}+\int_{t_{0}}^{t} \frac{A\left(\delta_{1}\right) x_{0}\left(\delta_{1}\right) d \delta_{1}}{\left(\delta_{1}-t_{0}\right)^{1-\alpha}}, \\
& \vdots \\
x_{k}(t)= & x_{0}+I_{\alpha}^{t_{0}}\left(A(t) x_{k-1}(t)\right) \\
= & x_{0}+\int_{t_{0}}^{t} \frac{A\left(\delta_{1}\right) x_{k-1}\left(\delta_{1}\right) d \delta_{1}}{\left(\delta_{1}-t_{0}\right)^{1-\alpha}},
\end{aligned}
$$

or we can write

$$
\begin{aligned}
x_{k}(t)= & x_{0}+I_{\alpha}^{t_{0}} A(t) x_{0} \\
& +I_{\alpha}^{t_{0}} A(t) I_{\alpha}^{t_{0}} A\left(\delta_{1}\right) x_{0}+\cdots \\
& +I_{\alpha}^{t_{0}} A(t) I_{\alpha}^{t_{0}} A\left(\delta_{1}\right) \cdots I_{\alpha}^{t_{0}} A\left(\delta_{k-1}\right) x_{0} \\
= & x_{0}+\int_{t_{0}}^{t} \frac{A\left(\delta_{1}\right) x_{0}}{\left(\delta_{1}-t_{0}\right)^{1-\alpha}} d \delta_{1} \\
& +\int_{t_{0}}^{t} \frac{A\left(\delta_{1}\right)}{\left(\delta_{1}-t_{0}\right)^{1-\alpha}} \int_{t_{0}}^{\delta_{1}} \frac{A\left(\delta_{2}\right) x_{0}}{\left(\delta_{2}-t_{0}\right)^{1-\alpha}} d \delta_{2} d \delta_{1}+\cdots \\
& +\int_{t_{0}}^{t} \frac{A\left(\delta_{1}\right)}{\left(\delta_{1}-t_{0}\right)^{1-\alpha}} \int_{t_{0}}^{\delta_{1}} \frac{A\left(\delta_{2}\right)}{\left(\delta_{2}-t_{0}\right)^{1-\alpha}} \\
& \cdots \int_{t_{0}}^{\delta_{k-1}} \frac{A\left(\delta_{k}\right) x_{0}}{\left(\delta_{k}-t_{0}\right)^{1-\alpha}} d \delta_{k} d \delta_{k-1} \cdots d \delta_{1} .
\end{aligned}
$$

So, using all of these calculations, the Peano-Baker series becomes

$$
\begin{aligned}
\Psi(t, \tau) & \\
= & +\int_{\tau}^{t} \frac{A\left(\delta_{1}\right)}{\left(\delta_{1}-t_{0}\right)^{1-\alpha}} d \delta_{1} \\
& +\int_{\tau}^{t} \frac{A\left(\delta_{1}\right)}{\left(\delta_{1}-t_{0}\right)^{1-\alpha}} \int_{\tau}^{\delta_{1}} \frac{A\left(\delta_{2}\right)}{\left(\delta_{2}-t_{0}\right)^{1-\alpha}} d \delta_{2} d \delta_{1}+\cdots \\
& +\int_{\tau}^{t} \frac{A\left(\delta_{1}\right)}{\left(\delta_{1}-t_{0}\right)^{1-\alpha}} \int_{\tau}^{\delta_{1}} \frac{A\left(\delta_{2}\right)}{\left(\delta_{2}-t_{0}\right)^{1-\alpha}} \cdots \int_{\tau}^{\delta_{k-1}} \frac{A\left(\delta_{k}\right)}{\left(\delta_{k}-t_{0}\right)^{1-\alpha}} d \delta_{k} d \delta_{k-1} \cdots d \delta_{1} \cdots .
\end{aligned}
$$

Definition 8 System (30) is said to be controllable if one can reach any state from any admissible initial state and initial control.

In simple words, we can say that a given system (30) is controllable on $\left[t_{0}, t_{f}\right]$ if given any initial state $X\left(t_{0}\right)=X_{0}$ there exists a continuous control signal $F(t)$ such that the corresponding solution of system (30) satisfies $X\left(t_{f}\right)=0$. 
Theorem 10 The linear conformable state equation (30) is controllable on $\left[t_{0}, t_{f}\right]$ if and only if the $n \times n$ matrix

$$
\begin{aligned}
G_{\alpha}\left(t_{0}, t_{f}\right) & =I_{\alpha}^{t_{0}, t_{f}}\left(e^{A \frac{\left(t-t_{0}\right)^{\alpha}}{\alpha}} B B^{T} e^{A^{T} \frac{\left(t-t_{0}\right)^{\alpha}}{\alpha}}\right) \\
& =\int_{t_{0}}^{t_{f}} e^{A \frac{\left(t-t_{0}\right)^{\alpha}}{\alpha}} B B^{T} e^{A^{T} \frac{\left(t-t_{0}\right)^{\alpha}}{\alpha}}\left(t-t_{0}\right)^{\alpha-1} d t,
\end{aligned}
$$

is invertible.

Proof Suppose $W\left(t_{0}, t_{f}\right)$ is invertible. Then given an $n \times 1$ vector $X_{0}$ choose

$$
F(t)=-B^{T} e^{A^{T} \frac{\left(t-t_{0}\right)^{\alpha}}{\alpha}} G_{\alpha}^{-1}\left(t_{0}, t\right) x_{0}, \quad t \in\left[t_{0}, t_{f}\right] .
$$

Then the control signal is continuous for $t \in\left[t_{0}, t_{f}\right]$ and the corresponding solution of (30) with $x\left(t_{0}\right)=x_{0}$ can be written as

$$
x(t)=e^{A \frac{\left(t-t_{0}\right)^{\alpha}}{\alpha}} x_{0}+\int_{t_{0}}^{t} e^{A \frac{\left(t-t_{0}\right)^{\alpha}}{\alpha}} e^{-A \frac{\left(\delta-t_{0}\right)^{\alpha}}{\alpha}} B F(\delta)\left(\delta-t_{0}\right)^{\alpha-1} d \delta .
$$

At $t=t_{f}$, we can write

$$
x\left(t_{f}\right)=e^{A \frac{\left(t_{f}-t_{0}\right)^{\alpha}}{\alpha}} x_{0}+e^{A \frac{\left(t_{f}-t_{0}\right)^{\alpha}}{\alpha}} \int_{t_{0}}^{t_{f}} e^{-A \frac{\left(\delta-t_{0}\right)^{\alpha}}{\alpha}} B F(\delta)\left(\delta-t_{0}\right)^{\alpha-1} d \delta .
$$

Consider that there exists $F(t)$ of the following form:

$$
F(t)=-B^{T} e^{A^{T} \frac{\left(t-t_{0}\right)^{\alpha}}{\alpha}} G_{\alpha}^{-1}\left(t_{0}, t\right) x_{0}, \quad t \in\left[t_{0}, t_{f}\right] .
$$

Then equation (34) becomes

$$
x\left(t_{f}\right)=e^{A \frac{\left(t_{f}-t_{0}\right)^{\alpha}}{\alpha}} x_{0}-e^{A \frac{\left(t_{f}-t_{0}\right)^{\alpha}}{\alpha}} \int_{t_{0}}^{t_{f}} e^{-A \frac{\left(\delta-t_{0}\right)^{\alpha}}{\alpha}} B B^{T} e^{-A \frac{\left(\delta-t_{0}\right)^{\alpha}}{\alpha}} W^{-1}\left(t_{0}, t_{f}\right) x_{0} d \delta,
$$

or

$$
\begin{aligned}
x\left(t_{f}\right)= & e^{A \frac{\left(t_{f}-t_{0}\right)^{\alpha}}{\alpha}} x_{0} \\
& -e^{A \frac{\left(t_{f}-t_{0}\right)^{\alpha}}{\alpha}}\left(\int_{t_{0}}^{t_{f}} e^{-A \frac{\left(\delta-t_{0}\right)^{\alpha}}{\alpha}} B B^{T} e^{-A^{T} \frac{\left(\delta-t_{0}\right)^{\alpha}}{\alpha}}\left(\delta-t_{0}\right)^{\alpha-1} d \delta\right) W^{-1}\left(t_{0}, t_{f}\right) x_{0}, \\
= & e^{A \frac{\left(t_{f}-t_{0}\right)^{\alpha}}{\alpha}} x_{0}-e^{A \frac{\left(t_{f}-t_{0}\right)^{\alpha}}{\alpha}} G\left(t_{0}, t_{f}\right) G^{-1}\left(t_{0}, t_{f}\right) x_{0}, \\
= & e^{A \frac{\left(t_{f}-t_{0}\right)^{\alpha}}{\alpha}} x_{0}-e^{A \frac{\left(t_{f}-t_{0}\right)^{\alpha}}{\alpha}} x_{0}, \\
= & 0 .
\end{aligned}
$$

Thus the conformable fractional linear state equation (30) is controllable.

For the converse claim, assume that (30) is controllable on $\left[t_{0}, t_{f}\right]$ and the Gramian matrix $G_{\alpha}\left(t_{0}, t_{f}\right)$ is not invertible. Since $G_{\alpha}\left(t_{0}, t_{f}\right)$ is not invertible then there exists a nonzero 
$n \times 1$ vector $x_{a}$ such that

$$
\begin{aligned}
& x_{a}^{T} G_{\alpha}\left(t_{0}, t_{f}\right) x_{a}=0, \\
& \int_{t_{0}}^{t_{f}} x_{a}^{T} e^{A \frac{\left(t-t_{0}\right)^{\alpha}}{\alpha}} B B^{T} e^{A^{T} \frac{\left(t-t_{0}\right)^{\alpha}}{\alpha}} x_{a}\left(t-t_{0}\right)^{\alpha-1} d t=0 .
\end{aligned}
$$

As the integrand in this expression is a nonnegative and continuous function $\left\|x_{a}^{T} e^{A \frac{\left(t-t_{0}\right)^{\alpha}}{\alpha}} B\right\|^{2}\left(t-t_{0}\right)^{\alpha-1}$, it follows that

$$
0=x_{a}^{T} e^{A \frac{\left(t-t_{0}\right)^{\alpha}}{\alpha}} B
$$

Since the conformable state equation is controllable on $\left[t_{0}, t_{f}\right]$, choosing $X_{0}=X_{a}$, there exists a continuous control vector $F(t)$ such that

$$
\begin{aligned}
0 & =e^{A \frac{\left(t_{f}-t_{0}\right)^{\alpha}}{\alpha}} x_{a}+e^{A \frac{\left(t_{f}-t_{0}\right)^{\alpha}}{\alpha}} \int_{t_{0}}^{t_{f}} e^{-A \frac{\left(\delta-t_{0}\right)^{\alpha}}{\alpha}} B F(\delta)\left(\delta-t_{0}\right)^{\alpha-1} d \delta \\
& =e^{A \frac{\left(t_{f}-t_{0}\right)^{\alpha}}{\alpha}}\left(x_{a}+\int_{t_{0}}^{t_{f}} e^{-A \frac{\left(\delta-t_{0}\right)^{\alpha}}{\alpha}} B F(\delta)\left(\delta-t_{0}\right)^{\alpha-1} d \delta\right) .
\end{aligned}
$$

So, we can write

$$
X_{a}=-\int_{t_{0}}^{t_{f}} e^{-A \frac{\left(\delta-t_{0}\right)^{\alpha}}{\alpha}} B\left(\delta-t_{0}\right)^{\alpha-1} F(\delta) d \delta .
$$

Multiplying equation (36) by $x_{a}^{T}$ on both sides and using (35), we have

$$
x_{a}^{T} x_{a}=-\int_{t_{0}}^{t_{f}} x_{a}^{T} e^{-A \frac{\left(\delta-t_{0}\right)^{\alpha}}{\alpha}} B\left(\delta-t_{0}\right)^{\alpha-1} F(\delta) d \delta=0,
$$

and this contradicts the fact that $x_{a} \neq 0$.

In the above theorem, $G_{\alpha}\left(t_{0}, t_{f}\right)$ is called the conformable fractional controllability Gramian and it is positive semidefinite, that is,

$$
X_{a}^{T} G_{\alpha}\left(t_{0}, t_{f}\right) X_{a} \geq 0
$$

Corollary 2 The linear conformable state equation (30) is controllable on $\left[t_{0}, t_{f}\right]$ if and only if the $n \times n$ conformable fractional controllability Gramian matrix is positive definite.

Proof The proof follows from the above theorem.

Lemma 7 There exist analytic scalar functions $\lambda_{1}(t), \lambda_{2}(t), \ldots, \lambda_{n-1}(t)$ such that

$$
e^{A \frac{\left(t-t_{0}\right)^{\alpha}}{\alpha}}=\sum_{k=0}^{n-1} \lambda_{k}(t) A^{k}
$$


Proof We know that the solution of $T_{\alpha}^{t_{0}} x=A x$ with $X(0)=I$ is $e^{A \frac{\left(t-t_{0}\right)^{\alpha}}{\alpha}}$. So, we can prove that there exist analytic scalar functions $\lambda_{1}(t), \lambda_{2}(t), \ldots, \lambda_{n-1}(t)$ such that

$$
\sum_{k=0}^{n-1} T_{\alpha}^{t_{0}} \lambda_{k}(t) A^{k}=\sum_{k=0}^{n-1} \lambda_{k}(t) A^{k+1}, \quad \sum_{k=0}^{n-1} \lambda_{k}(0) A^{k}=I
$$

According to Cayley-Hamilton theorem,

$$
A^{n}=-a_{0} I-a_{1} A-\cdots-a_{n-1} A^{n-1}
$$

where $a_{0}, a_{1}, \ldots, a_{n-1}$ are the coefficients of characteristic polynomial of $A$. Then (38) implies that

$$
\begin{aligned}
& \sum_{k=0}^{n-1} T_{\alpha}^{t_{0}} \lambda_{k}(t) A^{k}=\sum_{k=0}^{n-2} \lambda_{k}(t) A^{k+1}-\sum_{k=0}^{n-1} a_{k} \lambda_{n-1}(t) A^{k} \\
& =\sum_{k=1}^{n-1} \lambda_{k-1}(t) A^{k}-\sum_{k=0}^{n-1} a_{k} \lambda_{n-1}(t) A^{k}-a_{0} \lambda_{n-1}(t) I, \\
& \sum_{k=0}^{n-1} \lambda_{k}(0) A^{k}=I
\end{aligned}
$$

By equating the coefficients of like powers of $A$, we get the time-invariant linear state equation

$$
T_{\alpha}^{t_{0}}\left[\begin{array}{c}
\lambda_{0}(t) \\
\lambda_{1}(t) \\
\vdots \\
\lambda_{n-1}(t)
\end{array}\right]=\left[\begin{array}{cccc}
0 & \cdots & 0 & -a_{0} \\
1 & \cdots & 0 & -a_{1} \\
\vdots & \ddots & \vdots & \vdots \\
0 & \cdots & 1 & -a_{n-1}
\end{array}\right]\left[\begin{array}{c}
\lambda_{0}(t) \\
\lambda_{1}(t) \\
\vdots \\
\lambda_{n-1}(t)
\end{array}\right],\left[\begin{array}{c}
\lambda_{0}(0) \\
\lambda_{1}(0) \\
\vdots \\
\lambda_{n-1}(0)
\end{array}\right]=\left[\begin{array}{c}
1 \\
0 \\
\vdots \\
0
\end{array}\right] .
$$

Thus there exists an analytic solution of this linear state equation, which shows that the existence of analytic scalar functions $\lambda_{1}(t), \lambda_{2}(t), \ldots, \lambda_{n-1}(t)$ satisfying (39), and hence (38). So, we can write

$$
e^{A \frac{\left(t-t_{0}\right)^{\alpha}}{\alpha}}=\sum_{k=0}^{n-1} \lambda_{k}(t) A^{k}
$$

For the necessary and sufficient condition, we also need a matrix of order $n \times n m$ of the following form:

$$
\left[\begin{array}{llll}
B & A B & \cdots & A^{n-1} B
\end{array}\right]
$$

Theorem 11 System (30) is controllable for $t \in\left[t_{0}, t_{f}\right]$ if and only if the $n \times n m$ controllability matrix satisfies the following condition:

$$
\operatorname{rank}\left[\begin{array}{llll}
B & A B & \cdots & A^{n-1} B
\end{array}\right]=n .
$$


Proof We will prove that the rank condition (41) fails if and only if the $n \times n m$ conformable fractional Gramian matrix

$$
W\left(t_{0}, t_{f}\right)=\int_{t_{0}}^{t_{f}} e^{A \frac{\left(t-t_{0}\right)^{\alpha}}{\alpha}} B B^{T} e^{A^{T} \frac{\left(t-t_{0}\right)^{\alpha}}{\alpha}}\left(t-t_{0}\right)^{\alpha-1} d t
$$

is not invertible. Assume that the rank condition (41) fails, then there exists a nonzero vector $x_{a}$ such that

$$
x_{a}^{T}\left[\begin{array}{llll}
B & A B & \cdots & A^{n-1} B
\end{array}\right]=0,
$$

implying that

$$
x_{a}^{T} A^{k} B=0, \quad \text { for all } k=0,1, \ldots, n-1 .
$$

Also, we can write

$$
x_{a}^{T} W\left(t_{0}, t_{f}\right)=\int_{t_{0}}^{t_{f}} x_{a}^{T} e^{A \frac{\left(t-t_{0}\right)^{\alpha}}{\alpha}} B B^{T} e^{A^{T} \frac{\left(t-t_{0}\right)^{\alpha}}{\alpha}}\left(t-t_{0}\right)^{\alpha-1} d t .
$$

Using Lemma 7, we can write

$$
\begin{aligned}
x_{a}^{T} W\left(t_{0}, t_{f}\right) & =\int_{t_{0}}^{t_{f}} x_{a}^{T} \sum_{k=0}^{n-1} \lambda_{k}(t) A^{k} B B^{T} e^{A^{T} \frac{\left(t-t_{0}\right)^{\alpha}}{\alpha}}\left(t-t_{0}\right)^{\alpha-1} d t \\
& =\int_{t_{0}}^{t_{f}} x_{a}^{T} \sum_{k=0}^{n-1} \lambda_{k}(t) x_{a}^{T} A^{k} B B^{T} e^{A^{T} \frac{\left(t-t_{0}\right)^{\alpha}}{\alpha}}\left(t-t_{0}\right)^{\alpha-1} d t .
\end{aligned}
$$

From equation (42), it is clear that

$$
x_{a}^{T} G_{\alpha}\left(t_{0}, t_{f}\right)=0 .
$$

This shows that the conformable fractional Gramian is invertible and, according to Theorem 10, it is clear that system (30) is uncontrollable.

Conversely, assume that the given system (30) is not controllable. Then from Theorem 10, the conformable fractional Gramian is not invertible, and so there exists a nonzero vector $x_{a}$ such that

$$
x_{a}^{T} G_{\alpha}\left(t_{0}, t_{f}\right) x_{a}=0 .
$$

This implies, as in the proof of Theorem 10, that

$$
0=x_{a}^{T} e^{A \frac{\left(t-t_{0}\right)^{\alpha}}{\alpha}} B, \quad t \in\left[t_{0}, t_{f}\right] .
$$

If we substitute $t=t_{0}$ in equation (43), we obtain $0=x_{a}^{T} B$ and, after taking conformable fractional derivative $k$ times and evaluating the result at $t=t_{0}$, we get

$$
x_{a}^{T} A^{k} B=0, \quad k=0,1,2, \ldots, n-1 .
$$


Therefore

$$
x_{a}^{T}\left[\begin{array}{llll}
B & A B & \cdots & A^{n-1} B
\end{array}\right]=0,
$$

proving that the rank condition (41) fails. This completes the proof.

Example 3 Consider the differential equation of an $L C$ circuit

$$
\frac{d^{2} q}{d t^{2}}+\frac{1}{L C} q(t)=\frac{e_{0}}{L C}
$$

where $L$ is inductance, $C$ is capacitance, $q(t)$ is current, and $e_{0}$ is a constant driving force. Let $\omega_{0}^{2}=\frac{1}{L C}$ be the natural angular frequency. Then the conformable fractional transform operator [17] is

$$
\frac{d^{2}}{d t^{2}}=\omega_{0}^{2(1-\alpha)} T_{\alpha}^{(2)}
$$

By using relation (45), equation (44) becomes

$$
T_{\alpha}^{(2)} q(t)+\omega_{0}^{2 \alpha} q(t)=e_{0} \omega_{0}^{2 \alpha} .
$$

We want to check whether the system is controllable or not.

For this, we introduce the state variables

$$
x_{1}=q, \quad x_{2}=T_{\alpha} q .
$$

Then we see that the state representation of equation (45) is

$$
T_{\alpha}\left[\begin{array}{l}
x_{1} \\
x_{2}
\end{array}\right]=\left[\begin{array}{cc}
0 & 1 \\
-\omega_{0}^{2 \alpha} & 0
\end{array}\right]\left[\begin{array}{l}
x_{1} \\
x_{2}
\end{array}\right]+\left[\begin{array}{c}
0 \\
\omega_{0}^{2 \alpha}
\end{array}\right] e_{0},
$$

where $A=\left[\begin{array}{cc}0 & 1 \\ -\omega_{0}^{2 \alpha} & 0\end{array}\right], B=\left[\begin{array}{c}0 \\ \omega_{0}^{2 \alpha}\end{array}\right]$, thus the controllability matrix becomes

$$
\left[\begin{array}{ll}
B & A B
\end{array}\right]=\left[\begin{array}{cc}
0 & \omega_{0}^{2 \alpha} \\
\omega_{0}^{2 \alpha} & 0
\end{array}\right] .
$$

Since $\omega_{0}^{2 \alpha}=\left(\frac{1}{L C}\right)^{\alpha} \neq 0$, controllability matrix (48) has full rank, that is,

$$
\operatorname{rank}\left[\begin{array}{ll}
B & A B
\end{array}\right]=2 .
$$

Hence, by Theorem 11, system (47) is controllable.

In the case of harmonic source with angular velocity $\omega$, the equation becomes

$$
\frac{d^{2} q}{d t^{2}}+\frac{1}{L C} q(t)=e_{0} \cos \omega t
$$

By using the relation (45), equation (49) becomes

$$
T_{\alpha}^{(2)} q(t)+\omega_{0}^{2 \alpha} q(t)=e_{0} \omega_{0}^{2(\alpha-1)} \cos \omega t .
$$


Now the conformable state representation of this equation is

$$
T_{\alpha}\left[\begin{array}{l}
x_{1} \\
x_{2}
\end{array}\right]=\left[\begin{array}{cc}
0 & 1 \\
-\omega_{0}^{2 \alpha} & 0
\end{array}\right]\left[\begin{array}{l}
x_{1} \\
x_{2}
\end{array}\right]+\left[\begin{array}{c}
0 \\
\omega_{0}^{2(\alpha-1)}
\end{array}\right] e_{0} \cos \omega t .
$$

Here $A=\left[\begin{array}{cc}0 & 1 \\ -\omega_{0}^{2 \alpha} & 0\end{array}\right], B=\left[\begin{array}{c}0 \\ \omega_{0}^{2(\alpha-1)}\end{array}\right]$, so the conformable controllability Gramian becomes

$$
\left[\begin{array}{ll}
B & A B
\end{array}\right]=\left[\begin{array}{cc}
0 & \omega_{0}^{2(\alpha-1)} \\
\omega_{0}^{2(\alpha-1)} & 0
\end{array}\right] .
$$

As $\omega_{0}^{2(\alpha-1)}=\left(\frac{1}{L C}\right)^{\alpha-1} \neq 0$, the conformable fractional Gramian has rank 2 . This implies that (50) is controllable.

\section{Conclusions}

In this article, we have studied the sequential conformable linear equations. Using the conformable fractional derivative approach, we have developed the conformable controllability Gramian matrix, the conformable fundamental matrix, and the conformable controllability matrix. Our results are innovative compared to all the previous results obtained in the conformable case and are application-based as well. They will be highly helpful for the researchers in the future.

\section{Acknowledgements}

This research was funded by the Deanship of Scientific Research at Princess Nourah bint Abdulrahman University through the Fast-Track Research Funding Program.

\section{Funding}

There is no funding available for this article.

\section{Availability of data and materials}

Data sharing is not applicable to this article as no data sets were generated or analyzed during the current study.

\section{Competing interests}

The authors declare that they have no competing interests.

Authors' contributions

All authors equally contributed this manuscript and approved the final version.

\section{Author details}

${ }^{1}$ Centre for Advanced Studies in Pure and Applied Mathematics, Bahauddin Zakariya University, Multan, Pakistan. ${ }^{2}$ Department of Mathematics and General Sciences, Prince Sultan University, Riyadh, Saudi Arabia. ${ }^{3}$ Department of Medical Research, China Medical University, Taichung 40402, Taiwan. ${ }^{4}$ Department of Computer Science and Information Engineering, Asia University, Taichung, Taiwan. ${ }^{5}$ Pakistan Institute of Engineering and Technology, Multan, Pakistan. ${ }^{6}$ Department of Mathematical Sciences, Faculty of Sciences, Princess Nourah bint Abdulrahman University, P.O. Box 84428, Riyadh 11671, Saudi Arabia.

\section{Publisher's Note}

Springer Nature remains neutral with regard to jurisdictional claims in published maps and institutional affiliations.

Received: 24 May 2020 Accepted: 13 August 2020 Published online: 27 August 2020

\section{References}

1. Abdeljawad, T.: On conformable fractional calculus. Int. J. Comput. Appl. Math. 279, 57-66 (2015)

2. Abu Hammad, M., Khalil, R.: Abel's formula and Wronskian for conformable fractional differential equations. Int. J. Differ. Equ. Appl. 13(3), 177-183 (2014)

3. Al Horani, M., Abu Hammad, M., Khalil, R.: Variation of parameters for local fractional nonhomogeneous linear differential equations. J. Math. Comput. Sci. 16, 147-153 (2016)

4. Al-Mdallal, Q., Al-Refai, M., Syam, M., Sirhin, M.: Theoretical and computational perspectives on the eigenvalues of fourth order fractional Sturm-Liouville problem. Int. J. Comput. Math. 134(9), 461 (2019) 
5. Al-Mdallal, Q.M.: On fractional-Legendre spectral Galerkin method for fractional Sturm-Liouville problems. Chaos Solitons Fractals 116, 261-267 (2018)

6. Al-Mdallal, Q.M., Yusuf, H., Ali, A.: A novel algorithm for time-fractional foam drainage equation. Alex. Eng. J. 59(3), 1607-1612 (2020). https://doi.org/10.1016/j.aej.2020.04.007

7. Anderson, D.A., Camrud, E., Ulness, D.: On the nature of the conformable derivative and its applications to physics. J. Fract. Calc. Appl. 10(2), 92-135 (2019)

8. Atangana, A., Baleanu, D.: New fractional derivative with non-local and non-singular kernel: theory and application to heat transfer model. Therm. Sci. 20, 757 (2016)

9. Atangana, A., Baleanu, D., Alsaedi, A.: New properties of conformable derivative. Open Math. 13(1), 889-898 (2015)

10. Balachandran, K., Kokila, J.: On the controllability of fractional dynamical systems. Int. J. Appl. Math. Comput. Sci. 22(3), 523-531 (2012)

11. Birgani, O.T., Chandok, S., Dedović, N., Radenović, S.: A note on some recent results of the conformable derivative. Adv. Theory Nonlinear Anal. Appl. 3(1), 11-17 (2019)

12. Dalir, M., Bashour, M.: Applications of fractional calculus. Appl. Math. Sci. (Ruse) 4(21-24), 1021-1032 (2010)

13. Gokdogan, A., Unal, E., Celik, E.: Existence and uniqueness theorems for sequential linear conformable fractional differential equations. Miskolc Math. Notes 17(1), 267-279 (2016)

14. Kaczorek, T., Rogowski, K.: Fractional Linear Systems and Electrical Circuits. Studies in Systems, Decision and Control, vol. 13 (2014)

15. Khalil, R., Al Horani, M., Yousaf, A., Sababheh, M.: A new definition of fractional derivative. J. Comput. Appl. Math. 264, 65-70 (2014)

16. Kilbas, A.A., Srivastava, H.M., Trujillo, J.J.: Theory and Applications of Fractional Differential Equations. North-Holland Mathematics Studies, vol. 204. Elsevier, Amsterdam (2006)

17. Martinez, L., Rosales, J.J., Carreno, C.A., Lazano, J.M.: Electrical circuits described by fractional conformable derivative. Int. J. Circuit Theory Appl. 5(46), 1091-1100 (2018)

18. Matignon, D., d'Andréa-Novel, B.: Some results on controllability and observability of finite-dimensional fractional differential systems. In: Computational Engineering in Systems, IMACS, IEEE-SMC Lille, France, vol. 2, pp. 952-956 (1996)

19. Miller, K.S., Ross, B.: An Introduction to the Fractional Calculus and Fractional Differential Equations. A Wiley-Interscience Publication. Wiley, New York (1993)

20. Mirzae, F., Samadyar, N.: On the numerical method for solving a system of nonlinear fractional ordinary differential equations arising in HIV infection of $\mathrm{CD}_{4}^{+}$T cells. Iran. J. Sci. Technol. Trans. Sci. 43, 1127-1138 (2019)

21. Montseny, G., Audounet, J., Mbodje, B.: Optimal models of fractional integrators and application to systems with fading memory. Invited paper, IEEE/SMC' conference, Le Touquet France, Oct. (1993)

22. Muslim, M., Kumar, A.: Controllability of fractional differential equation of order $\alpha \in(1,2]$ with non-instantaneous impulses. Asian J. Control 20(2), 935-942 (2018)

23. Nanware, J.A., Dhaigude, D.B.: Existence and uniqueness of solutions of differential equations of fractional order with integral boundary conditions. J. Nonlinear Sci. Appl. 7(1), 246-254 (2014)

24. Patricio, M.F.S., Ramous, H., Patricio, M.: Solving initial and boundary value problems of fractional ordinary differential equations by using collocation and fractional powers. J. Comput. Appl. Math. 354, 348-359 (2019)

25. Podlubny, l.: Fractional Differential Equations. Math. Sci. Eng. Academic Press, San Diego (1999)

26. Podlubny, l.: Geometric and physical interpretation of fractional integration and fractional differentiation. Fract. Calc Appl. Anal. 5(4), 367-386 (2002)

27. Qureshi, S.: Effects of vaccination on measles dynamics under fractional conformable derivative with Liouville-Caputo operator. Eur. Phys. J. Plus 135, 63 (2020)

28. Rahmat, M.R.S.: A new definition of conformable fractional derivative on arbitrary time scales. Adv. Differ. Equ. 2019, 354 (2019)

29. Rough, W.J.: Linear System Theory, 2nd edn. Prentice Hall, Upper Saddle River (1996)

30. Unala, E., Gokdogan, A.: Solution of conformable fractional ordinary differential equation via differential tranasfer method. Optik 128, 264-273 (2016)

31. Unala, E., Gokdogan, A., Çelikc, E.: General solution to sequential linear conformable fractional differential equations with constant coefficients. arXiv:1602.01452v1 [math.CA]

32. Younus, A., Javaid, I., Zehra, A.: On controllability and observability of fractional continuous-time linear systems with regular pencils. Bull. Pol. Acad. Sci., Tech. Sci. 65(3), 297-304 (2017)

33. Zhao, D., Luo, M.: General conformable fractional derivative and its physical interpretation. Calcolo 54, 903-917 (2017)

34. Zhou, H.W., Yang, S., Zhang, S.Q.: Conformable derivative approach to anomalous diffusion. Phys. A, Stat. Mech. Appl. 491, 1001-1013 (2018) 